TRANSACTIONS OF THE

AMERICAN MATHEMATICAL SOCIETY

Volume 352, Number 11, Pages 4971-4987

S 0002-9947(00)02599-X

Article electronically published on June 20, 2000

\title{
STRONGLY ALMOST DISJOINT SETS AND WEAKLY UNIFORM BASES
}

\author{
Z. T. BALOGH, S. W. DAVIS, W. JUST, S. SHELAH, AND P. J. SZEPTYCKI
}

\begin{abstract}
A combinatorial principle CECA is formulated and its equivalence with GCH + certain weakenings of $\square_{\lambda}$ for singular $\lambda$ is proved. CECA is used to show that certain "almost point- $<\tau$ " families can be refined to point- $<\tau$ families by removing a small set from each member of the family. This theorem in turn is used to show the consistency of "every first countable $T_{1}$-space with a weakly uniform base has a point-countable base."
\end{abstract}

This research was originally inspired by the following question of Heath and Lindgren [4: Does every first countable Hausdorff space $X$ with a weakly uniform base have a point-countable base? The answer to this question is negative if MA + $\left(2^{\aleph_{0}}>\aleph_{2}\right)$ is assumed (see [2]). On the other hand, if CH holds and the space has at most $\aleph_{\omega}$ isolated points, then the answer is positive (see [1]). The starting point of this paper was the observation that if in addition to GCH also the combinatorial principle $\square_{\lambda}$ holds for every singular cardinal $\lambda$, then no bound on the number of isolated points is needed. An analysis of the proof led to the formulation of a combinatorial principle CECA. It turns out that CECA is equivalent to GCH + some previously known weakenings of $\square_{\lambda}$, but CECA has a different flavor than $\square_{\lambda}$-principles and may be easier to work with. The equivalence will be shown in Section 1.

In [3] it is shown that under certain conditions almost disjoint families $\left\{A_{\alpha}\right.$ : $\alpha<\kappa\}$ can be refined to disjoint families by removing small sets $A_{\alpha}^{\prime}$ from each $A_{\alpha}$. Let us say that a family $\left\{A_{\alpha}: \alpha<\kappa\right\}$ is point $-<\tau$ if for every $I \in[\kappa]^{\tau}$ the intersection $\bigcap_{\alpha \in I} A_{\alpha}$ is empty. In particular, a family is disjoint iff it is point- $<2$. In Section 2 the main theorem of this paper (Theorem 2) is derived from CECA. Roughly speaking, Theorem 2 asserts that certain families $\left\{A_{\alpha}: \alpha<\kappa\right\}$ that are "almost point- $<\tau$ " can be refined to point- $<\tau$ families by removing small sets $A_{\alpha}^{\prime}$ from each $A_{\alpha}$.

In Section 3, some related results for almost disjoint families are proved, and we explore how much of Theorem 2 can be derived from GCH alone rather than from CECA.

Received by the editors March 17, 1998.

2000 Mathematics Subject Classification. Primary 03E05, 03E35, 03E75, 54D70.

Key words and phrases. GCH, $\square$, strongly almost disjoint families, weakly uniform base, point countable base.

The first author's research was partially supported by NSF grant DMS-9623391. The third author's research was done during visits at Rutgers University and The Hebrew University, Jerusalem, which were supported by NSF grant DMS-9704477 and the Landau Center. The fourth author was partially supported by the Israel Basic Research Fund. This is publication number 674 in Shelah's publication list. 
In Section 4 we show that the positive answer to the question of [2], and more, follows already from Theorem 2 .

\section{The Closed COntinuous $\in$-Chain AXIOM}

Definition 1. Let $\tau$ be a regular cardinal. A set $M$ is $\tau$-closed if $[M]<\tau \subset M$. We say that a set $M$ is weakly $\tau$-closed if for every $I \in[M]^{\tau}$ there exists $J \in[I]^{\tau}$ such that $[J]^{<\tau} \subset M$. We say that $M$ is weakly closed if $M$ is weakly $\tau$-closed for all regular $\tau$ (equivalently: for all regular $\tau \leq|M|$ ). An $\in$-chain is a sequence $\left\langle M_{\xi}: \xi<\alpha\right\rangle$ such that $M_{\xi} \subset M_{\eta}$ and $\left\langle M_{\xi}: \xi \leq \eta\right\rangle \in M_{\eta+1}$ for all $\xi<\eta<\alpha$. The Closed Continuous $\in$-Chain Axiom for $\lambda$ and $\tau$ (abbreviated $\operatorname{CECA}_{\tau}(\lambda)$ ) is the following statement:

For every cardinal $\Theta>\lambda$, and for every pair of sets $A, B$ with $|A|=\lambda,|B|<$ $\lambda$, there exists a continuous $\in$-chain $\left\langle M_{\xi}\right\rangle_{\xi<c f(\lambda)}$ of weakly $\tau$-closed elementary submodels of $H(\Theta)$ such that

$-\left|M_{\xi}\right|<\lambda$ for every $\xi<c f(\lambda)$;

- $B \in M_{0} ;$ and

$-A \subset \bigcup_{\xi<c f(\lambda)} M_{\xi}$.

The Closed Continuous $\in$-Chain Axiom for $\lambda$ (abbreviated CECA $(\lambda)$ ) is obtained by requiring that the models $M_{\xi}$ in the definition of $\operatorname{CECA}_{\tau}(\lambda)$ are weakly closed. The Closed Continuous $\in$-Chain Axiom (abbreviated CECA) asserts that $\operatorname{CECA}(\lambda)$ holds for all uncountable cardinals $\lambda$.

Lemma 1. Let $\lambda$ be a regular uncountable cardinal. The following are equivalent:

(a) $\lambda^{<\lambda}=\lambda$.

(b) For every set $A$ with $|A| \leq \lambda$ there exists a weakly closed $M$ such that $A \subset M$ and $|M|=\lambda$.

(c) There exists a weakly $\lambda$-closed $M$ such that $|M|=\lambda$.

Proof. (a) $\Rightarrow(\mathrm{b})$. Suppose $\lambda^{<\lambda}=\lambda$, and $|A|=\lambda$. Build recursively an increasing sequence $\left\langle M_{\xi}: \xi<\lambda\right\rangle$ such that $A \subset M_{0},\left[M_{\xi}\right]^{<\lambda} \subset M_{\xi+1}$, and $\left|M_{\xi}\right|=\lambda$ for all $\xi<\lambda$. Let $M=\bigcup_{\xi<\lambda} M_{\xi}$. If $\tau \leq \lambda$ is regular, $I \in\left[M_{\xi}\right]^{\tau}$ and $K \in[I]^{<\tau}$, then $K \in\left[M_{\xi}\right]^{<\tau} \subseteq\left[M_{\xi}\right]^{<\lambda}$ for some $\xi<\lambda$, and hence $K \in M_{\xi+1} \subseteq M$, as required.

The implications (b) $\Rightarrow$ (c) and (c) $\Rightarrow$ (a) are obvious.

\section{Corollary 1. $\mathrm{CECA} \Rightarrow \mathrm{GCH}$.}

Lemma 2. Assume GCH. If $\lambda$ is an uncountable limit cardinal or if $\lambda=\kappa^{+}$for some regular infinite cardinal $\kappa$, then $\operatorname{CECA}(\lambda)$ holds.

Proof. Let $\lambda$ be as above, let $\Theta>\lambda$, and let $A, B$ be such that $|A|=\lambda,|B|<\lambda$. In both of these cases we can construct a continuous $\in$-chain $\left\langle M_{\xi}\right\rangle_{\xi<c f(\lambda)}$ of elementary submodels of $H(\Theta)$ such that

$-\left|M_{\xi}\right|<\lambda$ for every $\xi<c f(\lambda)$;

- $\left|M_{\xi+1}\right|$ is regular and $\left[M_{\xi+1}\right]^{<\left|M_{\xi+1}\right|} \subset M_{\xi+1}$ for every $\xi<c f(\lambda)$;

$-B \in M_{0}$; and

$-A \subset \bigcup_{\xi<c f(\lambda)} M_{\xi}$.

It remains to show that if $\delta<c f(\lambda)$ is limit, then $M_{\delta}$ is weakly closed. So assume $\delta<c f(\lambda)$ is limit, let $\tau$ be a regular cardinal, and let $I \in\left[M_{\delta}\right]^{\tau}$.

If $c f(\delta)<\tau$, then there is $\xi<\tau$ with $\left|M_{\xi} \cap I\right|=\tau$. Fix such $\xi$, and let $J=I \cap M_{\xi+1}$. Then $[J]^{<\tau} \subset\left[M_{\xi+1}\right]^{<\tau} \subseteq\left[M_{\xi+1}\right]^{<\left|M_{\xi+1}\right|} \subset M_{\xi+1} \subset M_{\delta}$. 
If $c f(\delta) \geq \tau$, then $[I]^{<\tau} \subset \bigcup_{\xi<\delta} M_{\xi}=M_{\delta}$, and we can take $J=I$.

Thus GCH implies that $\operatorname{CECA}(\kappa)$ holds for all $\kappa$, except perhaps if $\kappa=\lambda^{+}$ for a singular strong limit cardinal $\lambda$. Fortunately, it turns out that in this case $\operatorname{CECA}(\kappa)$ is equivalent to a weakening of $\square_{\lambda}$ that has been extensively studied by the fourth author. To prove the equivalence, let us introduce some notation.

We say that $\operatorname{Pr}(\lambda, \tau)$ holds if there exists an increasing continuous chain $\bar{N}=$ $\left\langle N_{i}: i<\lambda^{+}\right\rangle$such that $\left|N_{i}\right|=\lambda$ for each $i<\lambda^{+}$, each $N_{i}$ is weakly $\tau$-closed, and $\lambda^{+} \subset \bigcup_{i<\lambda^{+}} N_{i}$.

We say that $\operatorname{Pr}^{\prime}(\lambda, \tau)$ holds if there exists an increasing continuous chain $\bar{N}=$ $\left\langle N_{i}: i<\lambda^{+}\right\rangle$such that $\left|N_{i}\right|=\lambda$ for each $i<\lambda^{+}$, each $N_{i}$ is weakly $\tau$-closed with respect to sets of ordinals, and $\lambda^{+} \subset \bigcup_{i<\lambda^{+}} N_{i}$.

Note that if $2^{\lambda}=\lambda^{+}$, then in $\operatorname{Pr}(\lambda, \tau)$ and $\operatorname{Pr}^{\prime}(\lambda, \tau)$ we can demand that $\bigcup_{i<\lambda^{+}} N_{i}=H\left(\lambda^{+}\right)$.

In Definition 1.4 of [3], the following principle $S p(\sigma, \lambda)$ was introduced 1 There exists a sequence $\left\langle\mathcal{P}_{\xi}: \xi<\lambda^{+}\right\rangle$such that for all $\xi<\lambda^{+}$we have $\mathcal{P}_{\xi} \subset[\xi]^{\sigma}$ and $\left|\mathcal{P}_{\xi}\right| \leq \lambda$; moreover, if $\xi<\lambda^{+}$with $\sigma^{+}=c f(\xi)$ and $x$ is a cofinal subset of $\xi$ of cardinality $\sigma^{+}$, then $x$ can be written in the form $x=\bigcup\left\{x_{\nu}: \nu \in \sigma\right\}$, where for each $\nu \in \sigma$ we have $\left[x_{\nu}\right]^{\sigma} \subset \bigcup_{\eta<\xi} \mathcal{P}_{\eta}$.

The ideal $I[\kappa]$ was defined in [5] and [6]. We use here two equivalent definitions of $I[\kappa]$ given in [7].

Definition 2. For a regular uncountable cardinal $\kappa$, let $I[\kappa]$ be the family of all sets $A \subseteq \kappa$ such that the set $\{\delta \in A: \delta=c f(\delta)\}$ is not stationary in $\kappa$ and for some $\left\langle\mathcal{P}_{\alpha}: \alpha<\kappa\right\rangle$ we have:

(a) $\mathcal{P}_{\alpha}$ is a family of $<\kappa$ subsets of $\alpha$; and

(b) for every limit $\alpha \in A$ such that $c f(\alpha)<\alpha$ there is $x \subset \alpha$ such that $\operatorname{otp}(x)<$ $\alpha=\sup x$ and $\forall \beta<\alpha\left(x \cap \beta \in \bigcup_{\gamma<\alpha} \mathcal{P}_{\gamma}\right)$.

The following characterization of $I[\kappa]$ appears as Claim 1.2 in 7$]$. The abbreviation $\operatorname{nacc}(C)$ stands for "nonaccumulation points of $C$ " (in the order topology).

Lemma 3. Let $\kappa$ be a regular uncountable cardinal. Then $D \in I[\kappa]$ iff there exists a sequence $\left\langle C_{\beta}: \beta<\kappa\right\rangle$ such that:

(a) $C_{\beta}$ is a closed subset of $\beta$;

(b) if $\alpha \in \operatorname{nacc}\left(C_{\beta}\right)$ then $C_{\alpha}=C_{\beta} \cap \alpha$;

(c) for some club $E \subseteq \kappa$, for every $\delta \in D \cap E$ we have $\operatorname{cf}(\delta)<\delta$ and $\delta=\sup C_{\delta}$ and $\operatorname{otp}\left(C_{\delta}\right)=c f(\delta) ;$ and

(d) $\operatorname{nacc}\left(C_{\delta}\right)$ is a set of successor ordinals. $I\left[\lambda^{+}\right]$.

It is clear from the above lemma that Jensen's principle $\square_{\lambda}$ implies that $\lambda^{+} \in$

For $\tau \leq \lambda$, let $S_{\tau}^{\lambda^{+}}=\left\{\alpha<\lambda^{+}: \operatorname{cf}(\alpha)=\tau\right\}$.

Theorem 1. Let $\tau<\lambda$ be infinite cardinals with $\tau$ regular and $\lambda$ singular strong limit. The following are equivalent:

(a) $S_{\tau}^{\lambda^{+}} \in I\left[\lambda^{+}\right]$;

(b) $\operatorname{Pr}(\lambda, \tau)$;

\footnotetext{
${ }^{1}$ Actually, the principle introduced in 3 is more general and contains an extra parameter $\tau$, but the case $\tau=\sigma^{+}$is most relevant for the results in [3] and fits most neatly into the framework of the present paper.
} 
(c) $\operatorname{Pr}^{\prime}(\lambda, \tau)$;

(d) $\operatorname{CECA}_{\tau}\left(\lambda^{+}\right)$.

Moreover, if $\tau=\sigma^{+}$, then each of the above is also equivalent to:

(e) $S p(\sigma, \lambda)$.

Proof. The implications from (b) to (c) and from (d) to (b) are obvious

(c) $\Rightarrow$ (d). Let $\theta>\lambda^{+}$, and let $\bar{N}=\left\langle N_{i}: i<\lambda^{+}\right\rangle$exemplify $\operatorname{Pr}^{\prime}(\lambda, \tau)$. Let $\bar{M}=\left\langle M_{\xi}: \xi<\lambda^{+}\right\rangle$be an increasing continuous sequence such that $\bar{N} \in M_{0}$, and for all $\xi<\lambda^{+}$we have:

(1) $M_{\xi} \prec\left\langle H(\Theta),<^{*}\right\rangle$ (where $<^{*}$ is some wellorder relation on $H(\Theta)$ );

(2) $\left|M_{\xi}\right|=\lambda$; and

(3) $\bar{M} \mid(\xi+1) \in M_{\xi+1}$.

Let $M=\bigcup_{\xi<\lambda^{+}} M_{\xi}$. Note that (3) implies in particular that $\xi \in M_{\xi+1}$, and hence $\lambda^{+} \subset M$. Fix a bijection $h: M \rightarrow \lambda^{+}$such that $h \mid M_{\xi} \in M_{\xi+1}$ for all $\xi$. Such $h$ can be found by conditions (1) and (3): Recursively, let $h \mid M_{\xi+1}$ be the $<^{*}$ smallest bijection from $M_{\xi+1}$ onto an ordinal that extends $h \mid M_{\xi}$. For every $\xi<\lambda^{+}$, let $\eta(\xi)$ be the smallest ordinal $\eta \geq \xi$ such that $h\left[M_{\eta}\right]=\eta=M_{\eta} \cap \lambda^{+}=N_{\eta} \cap \lambda^{+}$. By $(2), \eta(\xi)<\lambda^{+}$for all $\xi$. Define

$$
E=\left\{\delta \in \mathbf{L I M} \cap \lambda^{+}: \forall \xi<\delta(\eta(\xi)<\delta)\right\} .
$$

Then $E$ is a closed unbounded subset of $\lambda^{+}$that consists of fixed points of the function $\eta$. Let $\left\{\delta_{\varepsilon}: \varepsilon<\lambda^{+}\right\}$be the increasing continuous enumeration of $E$.

We show that $\left\langle M_{\delta_{\varepsilon}}: \varepsilon<\lambda^{+}\right\rangle$witnesses $\operatorname{CECA}_{\tau}\left(\lambda^{+}\right)$. Let $\varepsilon<\lambda$ and let $I \in\left[M_{\delta_{\varepsilon}}\right]^{\tau}$. Note that we have $h[I] \in\left[\delta_{\varepsilon}\right]^{\tau}$ and $M_{\delta_{\varepsilon}} \cap \lambda^{+}=N_{\delta_{\varepsilon}} \cap \lambda^{+}$. So there is $J \subseteq h[I]$ with $|J|=\tau$ and $[J]^{<\tau} \subseteq N_{\delta_{\varepsilon}} \cap \mathbf{O N}$. We distinguish two cases:

Case 1: There exists $J^{\prime} \in[J]^{\tau}$ with $\sup J^{\prime}<\delta_{\varepsilon}$.

Let $\alpha=\eta\left(\sup J^{\prime}\right)$. Then $\alpha<\delta_{\varepsilon}, M_{\alpha} \cap \lambda=\alpha=h\left[M_{\alpha}\right]$, and $M_{\alpha} \cap \lambda^{+}=$ $N_{\alpha} \cap \lambda^{+}$. Thus, by shrinking $J^{\prime}$ if necessary, we may assume that $\left[J^{\prime}\right]^{<\tau} \subset N_{\alpha}$. Let $I^{\prime}=h^{-1} J^{\prime}$. Then $I^{\prime} \in[I]^{\tau}$. Moreover, if $K \in\left[I^{\prime}\right]^{<\tau}$, then $K=h^{-1} L$ for some $L \in\left[J^{\prime}\right]^{<\tau}$. Since $L, h \mid M_{\alpha} \in M_{\alpha+1}$ and $L \subset M_{\alpha+1}$, it follows that $K \in M_{\alpha+1} \subset M_{\delta_{\varepsilon}}$, as desired.

Case 2: There is no $J^{\prime}$ as in Case 1.

Then let $I^{\prime}=h^{-1} J$. Note that we must have $\operatorname{otp}(J)=\tau$ and $\sup J=\delta_{\varepsilon}$. Thus, if $K \in\left[I^{\prime}\right]^{<\tau}$, then $K=h^{-1} L$, where $L \in N_{\alpha}$ for some $\alpha<\delta_{\varepsilon}$ with $N_{\alpha}=M_{\alpha} \cap H\left(\lambda^{+}\right)$. Thus, arguing as in the previous case, one can show that $K \in M_{\alpha+1}$ and hence $\left[I^{\prime}\right]^{<\tau} \subset M_{\delta_{\varepsilon}}$.

(c) $\Rightarrow$ (a). Let $\bar{N}=\left\langle N_{\alpha}: \alpha<\lambda^{+}\right\rangle$be a sequence that witnesses $\operatorname{Pr}^{\prime}(\lambda, \tau)$. By thinning out the chain if necessary, we may assume that $\alpha \subset N_{\alpha}$ for each $\alpha<\lambda^{+}$. For each $\alpha$, let $\mathcal{P}_{\alpha}=N_{\alpha} \cap \mathcal{P}(\alpha)$. We claim that the sequence $\left\langle\mathcal{P}_{\alpha}: \alpha<\lambda^{+}\right\rangle$ witnesses that $S_{\tau}^{\lambda^{+}} \in I\left[\lambda^{+}\right]$. Condition (a) of Definition 2 is obvious. To verify that (b) also holds, let $\alpha \in S_{\tau}^{\lambda^{+}} \backslash\{\tau\}$. Pick a subset $I$ of $\alpha$ of order type $\tau$ such that $\sup I=\alpha$. Then $I \in\left[N_{\alpha} \cap \mathbf{O N}\right]^{\tau}$, and there exists $J \in[I]^{\tau}$ such that $[J]^{<\tau} \subset N_{\alpha}$. Since $\alpha$ is a limit ordinal and the sequence $\bar{N}$ is continuous, the latter implies that $[J]^{<\tau} \subset \bigcup_{\beta<\alpha} \mathcal{P}_{\beta}$. On the other hand, regularity of $\tau$ implies that $\operatorname{otp}(J)=\tau<\alpha$ and for every $\beta<\alpha$ the set $J \cap \beta$ has cardinality $<\tau$. Thus condition (b) holds, and we have shown that $S_{\tau}^{\lambda^{+}} \in I\left[\lambda^{+}\right]$.

(a) $\Rightarrow$ (d). We will actually prove something slightly more general which will allow us to show that the $M$ sequence works for several $\tau$ 's simultaneously. Assume 
$S_{\tau}^{\lambda^{+}} \subset D \in I\left[\lambda^{+}\right]$. Let $\left\langle C_{\alpha}: \alpha<\lambda\right\rangle$ and $E$ be witnesses that $D \in I\left[\lambda^{+}\right]$as in Lemma 3. Let $\mu=c f(\lambda)$, and let $\left(\kappa_{i}\right)_{i<\mu}$ be a sequence of cardinals with supremum $\lambda$ and such that $\max \{\mu, \tau\}<\kappa_{0}$ and $2^{\kappa_{i}} \leq \kappa_{i+1}$ for all $i<\mu$. Let $A, B$ be as in the assumptions of $\operatorname{CECA}_{\tau}\left(\lambda^{+}\right)$, and let $\Theta>\lambda^{+}$. Recursively construct a double sequence $\left\langle M_{\alpha}^{i}: \alpha<\lambda^{+}, i<\mu\right\rangle$ such that conditions 1-8 below are satisfied. For $\alpha<\lambda^{+}$, let $M_{\alpha}=\bigcup_{i<\mu} M_{\alpha}^{i}$.

1. $M_{\alpha} \prec H(\Theta)$;

2. $B \subset M_{0}$;

3. $A \subset \bigcup_{\alpha<\lambda^{+}} M_{\alpha}$;

4. the sequence $\left\langle M_{\alpha}: \alpha<\lambda^{+}\right\rangle$is continuous and increasing;

5. $\left\langle M_{\beta}: \beta \leq \alpha\right\rangle \in M_{\alpha+1}$;

6. $\left|M_{\alpha}^{i}\right|=\kappa_{i}$ and $M_{\alpha}^{i} \subset M_{\alpha}^{j}$ for $i<j$;

7. $\mathcal{P}\left(M_{\alpha+1}^{i}\right) \subset M_{\alpha+1}^{i+1}$;

8. if $\left|C_{\alpha}\right|<\lambda$, then $\mathcal{P}\left(\bigcup_{\beta \in C_{\alpha}} M_{\beta}^{i}\right) \subset M_{\alpha+1}$.

The construction is straightforward. Note that condition 8 can be satisfied since $\left|\bigcup_{\beta \in C_{\alpha}} M_{\beta}^{i}\right|=\left|C_{\alpha}\right| \cdot \kappa_{i}$, and $\left|C_{\alpha}\right|<\lambda$ for the relevant $\alpha$ 's.

Now let $\left\langle\alpha_{\xi}: \xi<\lambda^{+}\right\rangle$be the continuous increasing enumeration of $E$. We show that the sequence $\left\langle M_{\alpha_{\xi}}: \xi\left\langle\lambda^{+}\right\rangle\right.$witnesses $\operatorname{CECA}_{\tau}\left(\lambda^{+}\right)$. For this it suffices to verify that each $M_{\alpha_{\xi}}$ is weakly $\tau$-closed; the remaining requirements of $\operatorname{CECA}_{\tau}\left(\lambda^{+}\right)$ are already covered by conditions $1-6$. If $\alpha_{\xi}=\beta+1$ for some $\beta$, then $M_{\alpha_{\xi}}$ is weakly $\tau$-closed by condition 7 . So consider the case when $\alpha_{\xi}$ is a limit ordinal, and let $I \in\left[M_{\alpha_{\xi}}\right]^{\tau}$. We distinguish three cases:

Case 1: $c f\left(\alpha_{\xi}\right)=\tau \neq \mu$.

Then there exist $J \in[I]^{\tau}$ and $i<\mu$ such that $J \subset \bigcup_{\beta \in C_{\alpha_{\xi}}} M_{\beta}^{i}$. We will show that $[J]^{<\tau} \subset M_{\alpha_{\xi}}$. Let $K \in[J]^{<\tau}$. Then $K \subset \bigcup_{\beta \in C_{\alpha_{\xi}} \cap \gamma} M_{\beta}^{i}$ for some $\gamma \in C_{\alpha_{\xi}}$. Without loss of generality, we may assume that $\gamma \in$ nacc $C_{\alpha_{\xi}}$. Then $C_{\gamma}=C_{\alpha_{\xi}} \cap \gamma$. Since $\left|C_{\alpha_{\xi}}\right|=\tau<\lambda$, also $\left|C_{\gamma}\right|<\lambda$. By condition $8, K \in M_{\gamma+1}$, and hence $K \in M_{\alpha_{\xi}}$, as required.

Case 2: $c f\left(\alpha_{\xi}\right)=\tau=\mu$.

We will show that $[I]^{<\tau} \subset M_{\alpha_{\xi}}$. If $K \in[I]^{<\tau}$, then $K \subset M_{\beta+1}^{i}$ for some $\beta<\alpha_{\xi}$. By conditions 6 and $7, K \in M_{\beta+1}^{i+1} \subset M_{\alpha_{\xi}}$.

Case 3: $c f\left(\alpha_{\xi}\right) \neq \tau$.

Then there exists $\beta<\alpha_{\xi}$ such that $\left|I \cap M_{\beta+1}\right|=\tau$. Since $M_{\beta+1}$ is weakly $\tau$ closed and contained in $M_{\alpha_{\xi}}$, there exists $J \in\left[I \cap M_{\beta+1}\right]^{\tau}$ such that $[J]^{<\tau} \subset M_{\alpha_{\xi}}$.

(a) $\Rightarrow$ (e). Assume that $\tau=\sigma^{+}$, let $\left\langle M_{\alpha}^{i}: \alpha<\lambda^{+}, i<\mu\right\rangle$ be as in the previous part of the proof, i.e., such that conditions 1-8 hold, and let the sequence $\left\langle\alpha_{\xi}: \xi<\lambda^{+}\right\rangle$be defined as above. For each $\xi<\lambda^{+}$, let $\mathcal{P}_{\xi}=[\xi]^{\sigma} \cap M_{\xi+1}$.

Now suppose that $c f(\xi)=\tau$, and let $x \subset \xi$ be cofinal of cardinality $\tau$. We distinguish two cases:

Case 1: $\sigma<\mu$.

Then we can let $x_{\nu}=x$ for all $\nu<\sigma$. Each $y \in[x]^{\sigma}$ is contained in $M_{\alpha_{\eta}+1}^{i}$ for some $i<\mu$ and $\eta<\xi$. Thus $[x]^{\sigma} \subset \bigcup_{\eta<\xi} \mathcal{P}_{\eta}$, as required.

Case 2: $\sigma \geq \mu$.

Then let $x_{\nu}=x \cap \bigcup_{\beta \in C_{\alpha_{\xi}}} M_{\beta}^{\nu}$ for $\nu<\mu$ and $x_{\nu}=\emptyset$ for $\nu \geq \mu$. If $y \in\left[x_{\nu}\right]^{\sigma}$, then $y \subset \gamma \cap \bigcup_{\gamma \cap C_{\alpha_{\xi}}}$ for some $\gamma \in \operatorname{naccC}_{\alpha_{\xi}}$, and hence $y \in \mathcal{P}_{\eta}$ for some $\eta<\xi$ with $\gamma<\alpha_{\eta}$. 
(e) $\Rightarrow$ (a). Assume $\tau=\sigma^{+}$, and let $\left\langle\mathcal{P}_{\xi}: \xi<\lambda^{+}\right\rangle$witness that $S p(\sigma, \lambda)$ holds. For each $\xi$ let $\mathcal{R}_{\xi}$ be the union of $\mathcal{P}_{\xi}$ and the set of initial segments of elements of $\mathcal{P}_{\xi}$. Let $\xi \in S_{\tau}^{\lambda^{+}} \backslash\{\tau\}$. Now if $x \subset \xi$ is any cofinal subset of $\xi$ of order type $\tau$, then $x=\bigcup_{\nu \in \sigma} x_{\nu}$, where the family of all initial segments of $x_{\nu}$ is contained in $\bigcup_{\eta<\xi} \mathcal{R}_{\eta}$ for each $\nu<\sigma$. At least one of these $x_{\nu}$ 's must be cofinal in $\xi$ and of order type $\tau$, and this $x_{\nu}$ is exactly as required in Definition 2 .

Note that $\forall \tau \in \operatorname{Reg} \operatorname{CECA}_{\tau}(\kappa)$ does not always imply CECA $(\kappa)$. For example, if $2^{\aleph_{n}}=\aleph_{n+2}$ for all $n \in \omega$, then there are no weakly closed models of cardinality $\aleph_{n}$ for $n \in(0, \omega)$, and thus $\operatorname{CECA}\left(\aleph_{\omega}\right)$ fails. But if $\tau=\aleph_{n}$, then for each $m \geq n+1$ there are plenty of $\aleph_{n}$-closed models of cardinality $\aleph_{m}$, and thus $\operatorname{CECA}_{\aleph_{n}}\left(\aleph_{\omega}\right)$ holds. This anomaly cannot happen if $\kappa$ is a successor cardinal: If $\kappa=\lambda^{+}$for a regular $\lambda$, then $\mathrm{CECA}_{\lambda}(\kappa)$ implies the existence of weakly $\lambda$-closed models of cardinality $\lambda$, which in turn implies that $\lambda^{<\lambda}$. Now the proof of Lemma 2 can be adapted to derive $\operatorname{CECA}(\kappa)$. If $\kappa$ is the successor of a singular limit cardinal $\lambda$ and $\tau<\lambda$, then $\mathrm{CECA}_{\tau^{+}}(\kappa)$ implies that $2^{\tau} \leq \lambda$. Since for cofinally many $\tau$ we also will have $\tau>c f(\lambda)$, König's Theorem implies that $2^{\tau}$ is strictly less than $\lambda$. In other words, if $\mathrm{CECA}_{\tau}\left(\lambda^{+}\right)$holds and $\lambda$ is singular, then $\lambda$ must be a strong limit cardinal. For such $\lambda$ we have the following corollary to the proof of Theorem 1 .

Corollary 2. Let $\lambda$ be a singular strong limit cardinal. Then the following are equivalent:

(a) $\lambda^{+} \in I\left[\lambda^{+}\right]$

(b) $S_{\tau}^{\lambda^{+}} \in I\left[\lambda^{+}\right]$for all regular $\tau<\lambda$;

(c) $\operatorname{CECA}\left(\lambda^{+}\right)$;

(d) $\operatorname{CECA}_{\tau}\left(\lambda^{+}\right)$holds for all regular $\tau \in \lambda$.

Proof. The equivalence between (a) and (b) follows from Shelah's observation that $I\left[\lambda^{+}\right]$is a normal, and hence $\lambda^{+}$-complete, ideal (see [5], 6], or [7]). The equivalence between (b) and (d) was established in Theorem 2, The implication (c) $\Rightarrow(\mathrm{d})$ is

obvious. To see that (a) implies (c), note that the last part of the proof of Theorem2 shows that if $\lambda^{+} \in I\left[\lambda^{+}\right]$, then the sequence $\left\langle M_{\alpha_{\xi}}: \xi<\lambda^{+}\right\rangle$constructed from $D=\lambda^{+}$witnesses $\operatorname{CECA}_{\tau}\left(\lambda^{+}\right)$simultaneously for all $\tau \in \lambda \cap$ Reg.

\section{THE MAIN THEOREM}

Let $\tau$ be a cardinal. Recall that a sequence of sets $\left\langle A_{\alpha}\right\rangle_{\alpha<\kappa}$ is said to be point$<\tau$ at a point $a$ if $\left|\left\{\alpha: a \in A_{\alpha}\right\}\right|<\tau$. We will say that a sequence of sets is point $-<\tau$ if it is point- $<\tau$ at every point. Notice that $\left\langle A_{\alpha}\right\rangle_{\alpha<\kappa}$ is point $<\tau$ if for every $I \in[\kappa]^{\tau}$ the intersection $\bigcap_{\alpha \in I} A_{\alpha}$ is empty.

Theorem 2. Assume CECA. Suppose that $\sigma, \tau$ are regular infinite cardinals, and let $\left\langle A_{\alpha}\right\rangle_{\alpha<\kappa}$ be a sequence of (not necessarily distinct) sets such that one of the following conditions is satisfied:

(1.1) For every $I \in[\kappa]^{\tau}$ there is $J \in[I]^{<\tau}$ such that $\left|\bigcap_{\alpha \in J} A_{\alpha}\right|<\sigma$.

(1.2) For every $I \in[\kappa]^{\tau}$ there is a $J \in[I]^{<\tau}$ such that $\left|\bigcap_{\alpha \in J} A_{\alpha}\right| \leq \sigma$ and for each $S \in\left[\bigcup_{\alpha<\kappa} A_{\alpha}\right]^{\sigma}$ we have $\left|\left\{\alpha: S \subset A_{\alpha}\right\}\right| \leq \sigma$.

Then there exist $\left\langle A_{\alpha}^{\prime}\right\rangle_{\alpha<\kappa}$ such that $\left|A_{\alpha}^{\prime}\right| \leq \sigma$ for each $\alpha<\kappa$ and the sequence $\left\langle A_{\alpha} \backslash A_{\alpha}^{\prime}\right\rangle_{\alpha<\kappa}$ is point $-<\tau$. 
Proof. Suppose the theorem is false, let $\kappa$ be the smallest cardinal for which the theorem fails, and fix a counterexample $\bar{A}=\left\langle A_{\alpha}\right\rangle_{\alpha<\kappa}$ and $\sigma, \tau$ that witness this fact. Throughout the proof, let $\Theta$ denote a "sufficiently large" cardinal, and let $A=\bigcup_{\alpha<\kappa} A_{\alpha}$. In the proof we will consider all possible ways in which $\kappa, \sigma, \tau$ can be related to each other, and we will derive a contradiction in each case. To begin with, note that we may without loss of generality assume that $\tau \leq \kappa$; otherwise the conclusion of the theorem is vacuously true. Now let us eliminate the case $\sigma \geq \kappa$.

Lemma 4. Suppose that $M \prec H(\Theta)$ is weakly $\tau$-closed with respect to sets of ordinals, i.e., for every $I \in[M \cap \kappa]^{\tau}$ there exists $J \in[I]^{\tau}$ with $[J]^{<\tau} \subset M$. Moreover, suppose $\bar{A} \in M$ and $\sigma \subset M$. Then the sequence $\left\langle A_{\alpha} \backslash M\right\rangle_{\alpha \in \kappa \cap M}$ is point $-<\tau$.

Corollary 3. $\sigma<\kappa$.

Proof. Let $M \prec H(\Theta)$ be weakly closed and such that $\sigma \subset M,|M|=\sigma$, and $M$ contains everything relevant. For each $\alpha<\kappa$, let $A_{\alpha}^{\prime}=M$. If $\sigma \geq \kappa$, then $\kappa \cap M=\kappa$, and Lemma 4 implies the conclusion of Theorem 2.

Proof of Lemma 4. Suppose towards a contradiction that there are $a$ and $I \in$ $[\kappa \cap M]^{\tau}$ such that $a \in \bigcap_{\alpha \in I} A_{\alpha} \backslash M$. Since $M$ is weakly $\tau$-closed with respect to sets of ordinals 2 by passing to a subset of $I$ if necessary, we may assume that $[I]^{<\tau} \subset M$. Since (1.1) or (1.2) holds, we can find $J \in[I]^{<\tau}$ such that

$$
B=\left|\bigcap_{\alpha \in J} A_{\alpha}\right| \leq \sigma
$$

Since $\bar{A}, J \in M$, it follows that $B \in M$. Since $\sigma \subset M$ and $|B| \subset \sigma$, we conclude that $B \subset M$, which contradicts the assumption that $a \in B \backslash M$.

Now let us reveal how CECA will be used in the remainder of this proof.

Lemma 5. Suppose that there is a continuous increasing chain $\left\langle M_{\xi}\right\rangle_{\xi<c f(\kappa)}$ of weakly closed elementary submodels of $H(\Theta)$ such that $\left|M_{\xi}\right|<\kappa$ for each $\xi<c f(\kappa)$, that $M_{0}$ contains everything relevant, that $\sigma \subset M_{0}$, that $[\kappa]^{<c f(\kappa)} \subset \bigcup_{\xi<c f(\kappa)} M_{\xi}$, and that for every $\xi<\kappa$ :

(i) the sequence $\left\langle A_{\alpha} \backslash M_{\xi}\right\rangle_{\alpha \in M_{\xi} \cap \kappa}$ is point $-<\tau$;

(ii) $\alpha \in \kappa \backslash M_{\xi}$ implies $\left|M_{\xi} \cap A_{\alpha}\right| \leq \sigma$; and

(iii) if $\alpha \in M_{\xi}$ and $\left|A_{\alpha}\right| \leq\left|M_{\xi}\right|$, then $A_{\alpha} \subset M_{\xi}$.

Then there are $A_{\alpha}^{\prime} \in\left[A_{\alpha}\right] \leq \sigma$ for $\alpha<\kappa$ such that the sequence $\left\langle A_{\alpha} \backslash A_{\alpha}^{\prime}\right\rangle_{\alpha, \kappa}$ is point $-<\tau$.

Proof. Let the $M_{\xi}$ 's and $A_{\alpha}$ 's be as in the assumption. By the choice of $\kappa$ and (ii) we can pick, for every $\xi<\kappa$ and $\alpha \in \kappa \cap M_{\xi+1} \backslash M_{\xi}$, an $A_{\alpha}^{\prime} \in\left[A_{\alpha}\right] \leq \sigma$ in such a way that $A_{\alpha} \cap M_{\xi} \subset A_{\alpha}^{\prime}$ and the sequence $\left\langle A_{\alpha} \backslash A_{\alpha}^{\prime}\right\rangle_{\alpha \in \kappa \cap\left(M_{\xi+1} \backslash M_{\xi}\right)}$ is point- $<\tau$.

We claim that the sequence $\left\langle A_{\alpha} \backslash A_{\alpha}^{\prime}\right\rangle_{\alpha<\kappa}$ is point- $<\tau$. To see this, let $a \in A$, and let $M^{*}=\bigcup_{\xi<c f(\kappa)} M_{\xi}$.

Case 1: $a \in M^{*}$.

Then there exists a unique $\xi<c f(\kappa)$ such that $a \in M_{\xi+1} \backslash M_{\xi}$. For this $\xi$, the following hold:

\footnotetext{
${ }^{2}$ If $M$ has only the property that for every subset $I \in[M \cap \kappa]^{\tau^{+}}$there exists $J \in[I]^{\tau}$ with $[J]^{<\tau} \subset M$, then the argument presented here shows that the sequence $\left\langle A_{\alpha} \backslash M\right\rangle_{\alpha \in \kappa \cap M}$ is point$<\tau^{+}$.
} 
- By (i), $\left|\left\{\alpha \in \kappa \cap M_{\xi}: a \in A_{\alpha}\right\}\right|<\tau$.

- By construction, $\left|\left\{\alpha \in \kappa \cap\left(M_{\xi+1} \backslash M_{\xi}\right): a \in A_{\alpha} \backslash A_{\alpha}^{\prime}\right\}\right|<\tau$.

- If $\alpha \in \kappa \backslash M_{\xi+1}$, then $A_{\alpha} \cap M_{\xi+1} \subset A_{\alpha}^{\prime}$, and hence no $\alpha \in \kappa \backslash M_{\xi+1}$ satisfies $a \in A_{\alpha} \backslash A_{\alpha}^{\prime}$.

It follows that the sequence $\left\langle A_{\alpha} \backslash A_{\alpha}^{\prime}\right\rangle_{\alpha<\kappa}$ is point- $<\tau$ at $a$.

Case 2: $a \notin M^{*}$.

We show that $M^{*}$ is weakly $\tau$-closed with respect to sets of ordinals. Let $I \in$ $\left[M^{*} \cap \kappa\right]^{\tau}$. If $\tau=c f(\kappa)$, then $[\kappa]^{<\tau} \subset M^{*}$ and hence $[I]^{<\tau} \subset M^{*}$. If $\tau \neq c f(\kappa)$, then there exists $\xi<c f(\kappa)$ with $\left|M_{\xi} \cap I\right|=\tau$, and $J=M_{\xi} \cap I$ is as required. Now Lemma 4 implies that the sequence $\left\langle A_{\alpha} \backslash A_{\alpha}^{\prime}\right\rangle_{\alpha<\kappa}$ is point- $<\tau$ at $a$.

Since we may assume $\sigma<\kappa$, CECA clearly implies the existence of a sequence $\left\langle M_{\xi}\right\rangle_{\xi<c f(\kappa)}$ that satisfies all unnumbered assumptions of Lemma 5. By Lemma 4 this sequence will also satisfy condition (i). How can we make sure that condition (ii) also holds? This depends on $\tau$.

Lemma 6. Suppose that $M \prec H(\Theta)$ is weakly closed, $\bar{A} \in M$, and $\max \{\sigma, \tau\} \subset$ $M$. Then $\alpha \in \kappa \backslash M$ implies $\left|A_{\alpha} \cap M\right| \leq \sigma$.

Proof. Assume towards a contradiction that $\alpha \in \kappa \backslash M$ is such that $\left|A_{\alpha} \cap M\right| \geq \sigma^{+}$. Since $M$ is weakly closed 3 there exists $Z \in\left[A_{\alpha} \cap M\right]^{\sigma} \cap M$. We are going to prove that the set $I=\left\{\beta \in \kappa: Z \subset A_{\beta}\right\}$ has cardinality $>\max \{\sigma, \tau\}$, in contradiction with conditions (1.1) and (1.2) of Theorem [2. Suppose that $|I| \leq \max \{\sigma, \tau\}$. Note that since $\kappa, \bar{A}, Z \in M$, it follows that $I \in M$, and there is a one-to-one function $f \in M$ that maps $I$ into $\max \{\sigma, \tau\} \subset M$. It follows that $I \subset M$. On the other hand, $\alpha \in I \backslash M$, which gives a contradiction.

It follows from Lemma $\left[\right.$ that if $\tau<\kappa$, then we can find a sequence $\left\langle M_{\xi}\right\rangle_{\xi<c f(\kappa)}$ that satisfies the assumption of Lemma [5. So it remains to prove Theorem 2 for the case $\sigma<\tau=\kappa$. If $\tau$ happens to be equal to $\sigma^{+}$, then any chain we get from CECA will satisfy $\left|M_{\xi}\right| \leq \sigma$, and we get condition (ii) for free. If $\sigma^{+}<\tau=\kappa$, then we need to take advantage of GCH. The following lemma shows how to handle this last remaining case.

Lemma 7. Suppose $\kappa=\tau \geq \sigma^{++}$, and let $\left\langle M_{\xi}\right\rangle_{\xi<\kappa}$ be a continuous increasing sequence of elementary submodels of $H(\Theta)$ such that each $M_{\xi}$ has cardinality less than $\kappa$ and $[\kappa]^{<\kappa} \subset \bigcup_{\xi<\kappa} M_{\xi}$. Then there exists a continuous subsequence $\left\langle M_{\xi_{\nu}}\right\rangle_{\nu<\kappa}$ such that

$$
\nu<\kappa \text { and } \alpha \in \kappa \backslash M_{\xi_{\nu}} \text { imply }\left|A_{\alpha} \cap M_{\xi_{\nu}}\right| \leq \sigma .
$$

Proof. Let $A=\bigcup_{\alpha<\kappa} A_{\alpha}$. Note that since $\kappa \geq \tau$, conditions (1) in Theorem 2 imply that for every $S \in[A]^{\sigma}$ the set $h(S)=\left\{\alpha<\kappa: S \subset A_{\alpha}\right\}$ has cardinality $<\kappa$. Since $\kappa \geq \sigma^{++}$and $\kappa(=\tau)$ is regular, GCH implies that there is a continuous subsequence $\left\langle M_{\xi_{\nu}}\right\rangle_{\nu<\kappa}$ of $\left\langle M_{\xi}\right\rangle_{\xi<\kappa}$ such that

$$
\text { for every } \nu<\kappa \text { and every } S \in[A]^{\sigma} \cap M_{\xi_{\nu+1}} \text { we have } h(S) \subset M_{\xi_{\nu+1}} \text {. }
$$

We claim that $\left\langle M_{\xi_{\nu}}\right\rangle_{0<\nu<\kappa}$ is as required. Let $N_{\nu}$ denote $M_{\xi_{\nu}}$. Suppose towards a contradiction that $0<\mu<\kappa$ and $\alpha \in \kappa \backslash N_{\mu}$ are such that $\left|A_{\alpha} \cap N_{\mu}\right| \geq \sigma^{+}$. We distinguish two cases.

\footnotetext{
${ }^{3}$ Note that in this argument, as well as in the proof of the next lemma, only the following consequence of weak closedness is used: If $I \in[M]^{\sigma^{+}}$, then there is $J \in[I]^{\sigma}$ such that $J \in M$.
} 
Case 1: $\mu=\nu+1$ for some $\nu$.

Since $N_{\nu+1}$ is weakly closed, there exists $S \in\left[A_{\alpha}\right]^{\sigma} \cap N_{\nu+1}$. By $(4), h(S) \subset N_{\nu+1}$. Since $\alpha$ in $h(S)$, we have $\alpha \in N_{\nu+1}=N_{\mu}$, contradicting the choice of $\alpha$.

Case 2: $\mu$ is a limit ordinal.

We will show that

$$
\text { there are } \nu<\mu \text { and } S \in\left[A_{\alpha}\right]^{\sigma} \text { such that } S \in N_{\nu+1} \text {. }
$$

Indeed, since $N_{\nu}$ is weakly closed and $\left|A_{\alpha} \cap N_{\nu}\right| \geq \sigma^{+}$, we can pick $S \in\left[A_{\alpha}\right]^{\sigma} \cap N_{\nu}$. Since $N_{\nu}=\bigcup_{\nu<\mu} N_{\nu}$, there is $\nu<\mu$ such that $S \in N_{\nu+1}$. Now we can derive a contradiction as in the previous case.

\section{RELATED THEOREMS AND EXAMPLES}

We first present a modification of Theorem 2 exhibiting when we can obtain point- $<\tau$ families where $\tau$ is finite. Note that a family of sets is point- $<2$ if and only if it is pairwise disjoint.

Theorem 3. Assume CECA, and let $n \geq 1$. Suppose that $\sigma$ is a regular cardinal, and let $\left\langle A_{\alpha}\right\rangle_{\alpha<\kappa}$ be a sequence of (not necessarily distinct) sets such that $\left|A_{\alpha}\right| \leq \sigma^{+n}$ and one of the following conditions is satisfied:

(1.3) $\left|A_{\alpha} \cap A_{\beta}\right|<\sigma$ for all $\alpha<\beta<\kappa$;

(1.4) $\left|A_{\alpha} \cap A_{\beta}\right| \leq \sigma$ for all $\alpha<\beta<\kappa$, and for each $S \in\left[\bigcup_{\alpha<\kappa} A_{\alpha}\right]^{\sigma}$ $\left|\left\{\alpha: S \subset A_{\alpha}\right\}\right| \leq \sigma$.

Then there exist $\left\langle A_{\alpha}^{\prime}\right\rangle_{\alpha<\kappa}$ such that $\left|A_{\alpha}^{\prime}\right| \leq \sigma$ for each $\alpha<\kappa$ and the sequence $\left\langle A_{\alpha} \backslash A_{\alpha}^{\prime}\right\rangle_{\alpha<\kappa}$ is point $<n+1$.

Proof. First apply Theorem 2 for $\tau=\aleph_{0}$ to get sets $A_{\alpha}^{\prime \prime} \in\left[A_{\alpha}\right]^{\sigma}$ such that $\left\langle A_{\alpha} \backslash A_{\alpha}^{\prime \prime}\right\rangle_{\alpha<\kappa}$ is point-finite. The step from point-finite to point- $<n+1$ can be made in $\mathrm{ZFC}$ :

Lemma 8. Let $n \geq 1$. Suppose that $\sigma$ is a regular infinite cardinal, and also that $\left\{A_{\alpha}: \alpha<\kappa\right\}$ is a point-finite family of sets satisfying $\left|A_{\alpha}\right| \leq \sigma^{+n}$ for each $\alpha$ and $\left|A_{\alpha} \cap A_{\beta}\right| \leq \sigma$ for each pair of distinct $\alpha, \beta \in \kappa$. Then there are $A_{\alpha}^{\prime} \in\left[A_{\alpha}\right]^{\sigma}$ for each $\alpha$ such that $\left\{A_{\alpha} \backslash A_{\alpha}^{\prime}: \alpha<\kappa\right\}$ is point $<n+1$.

Proof of Lemma 8. First let us prove the lemma for the case $n=1$. Let $\kappa$ be the minimal cardinal such that there is a family of size $\kappa$ that forms a counterexample to the theorem. Clearly $\kappa>\sigma$. First assume that $\kappa>\sigma^{+}$. Fix an $\in$-chain of elementary submodels $\left\{M_{\xi}: \xi<c f(\kappa)\right\}$ such that

1. $\sigma^{+} \subseteq M_{0}$, and $\left|M_{\xi}\right|<\kappa$ for each $\xi<\kappa$,

2. $\left\{A_{\alpha}: \alpha<\kappa\right\} \in M_{0}$, and

3. $\left\{A_{\alpha}: \alpha<\kappa\right\} \subseteq \bigcup\left\{M_{\xi}: \xi<c f(\kappa)\right\}$.

Using the minimality of $\kappa$, for each $\xi \in c f(\kappa)$ fix a sequence

$$
\left\{A_{\alpha}^{\prime}: \alpha \in \kappa \cap\left(M_{\xi+1} \backslash M_{\xi}\right)\right\}
$$

of sets of size $\sigma$ such that $\left\{A_{\alpha} \backslash A_{\alpha}^{\prime}: \alpha \in \kappa \cap M_{\xi+1}\right\}$ is pairwise disjoint. Note that, by point-finiteness, if $\alpha \notin M_{\xi}$ then $A_{\alpha} \cap M_{\xi}=\emptyset$. Also $\sigma^{+} \subseteq M_{\xi}$ implies that $A_{\alpha} \subseteq M_{\xi}$ for each $\alpha \in M_{\xi}$. Therefore $\left\{A_{\alpha} \backslash A_{\alpha}^{\prime}: \alpha \in \kappa\right\}$ is pairwise disjoint.

The case for $\kappa=\sigma^{+}$is similar. Fix an $\in$-chain as above of elementary submodels $\left\{M_{\xi}: \xi<c f(\kappa)\right\}$ of size $\sigma$ so that $\sigma \subseteq M_{0}$, and let $A_{\alpha}^{\prime}=A_{\alpha} \cap M_{\xi+1}$, where $\alpha \in M_{\xi+1} \backslash M_{\xi}$. To see that $\left\{A_{\alpha} \backslash A_{\alpha}^{\prime}: \alpha \in \kappa\right\}$ is pairwise disjoint it suffices to 
observe that, as above, if $\alpha \notin M_{\xi}$ then $A_{\alpha} \cap M_{\xi} \neq \emptyset$ and, in addition, if $\alpha, \beta \in M_{\xi}$ then $A_{\alpha} \cap A_{\beta} \subseteq M_{\xi}$.

Now suppose $m>1$ and the lemma is true for all $n<m$. Let $\left\langle A_{\alpha}\right\rangle_{\alpha<\kappa}$ be a sequence of sets such that $\left|A_{\alpha}\right| \leq \sigma^{+m}$ for each $\alpha$ and $\left|A_{\alpha} \cap A_{\beta}\right| \leq \sigma$ for each pair of distinct $\alpha, \beta \in \kappa$. Let $\nu=\sigma^{+(m-1)}$. Then $\sigma^{+m}=\nu^{+}$, and hence the lemma for $n=1$ implies that there are $A_{\alpha}^{\prime \prime} \in\left[A_{\alpha}\right]^{\nu}$ for each $\alpha$ such that the family $\left\{A_{\alpha} \backslash A_{\alpha}^{\prime}: \alpha<\kappa\right\}$ is pairwise disjoint. Since the lemma is true for $n=m-1$, there are $A_{\alpha}^{\prime} \in\left[A_{\alpha}^{\prime \prime}\right]^{\sigma}$ such that the family $\left\{A_{\alpha}^{\prime \prime} \backslash A_{\alpha}^{\prime}: \alpha<\kappa\right\}$ is point- $<m$. Now we claim that the family $\left\{A_{\alpha} \backslash A_{\alpha}^{\prime}: \alpha<\kappa\right\}$ is point- $<m+1$. For any $a$, there are at most $m-1$ indices $\alpha$ such that $a \in A_{\alpha}^{\prime \prime} \backslash A_{\alpha}^{\prime}$. Moreover, the family $\left\{A_{\alpha} \backslash A_{\alpha}^{\prime \prime}: \alpha<\kappa\right\}$ is disjoint, so there is at most one index $\alpha$ with $a \in A_{\alpha} \backslash A_{\alpha}^{\prime \prime}$. So we are done.

The following example shows that Lemma 8 is in a sense the best possible.

Example 1. For each $n \in \omega$ there is a point-<n+1 family $\mathcal{A}_{n}$ of sets of size $\aleph_{n}$ such that

(a) $A \cap B$ is finite for each pair of distinct $A, B \in \mathcal{A}_{n}$, and

(b) $\mathcal{A}_{n}$ has no point- $<n$ refinement of the form $\left\{A \backslash A^{\prime}: A \in \mathcal{A}_{n}\right\}$ where each $A^{\prime}$ is countable.

Proof. For each $n \in \omega$, each $i \in n$ fix $s: n \backslash\{i\} \rightarrow \omega_{n}$ let $A_{s}=\left\{t \in{ }^{n} \omega_{n}\right.$ : $t$ extends $s\}$. Let $\mathcal{A}_{n}=\bigcup_{i \in n}\left\{A_{s}: s \in{ }^{n \backslash\{i\}} \omega_{n}\right\}$. We will show that $\mathcal{A}_{n}$ is the required family for each $n \in \omega$.

$(n=2): \mathcal{A}_{2}$ is the family of rows and columns in $\omega_{2} \times \omega_{2}$. Clearly this family is point- $<3$. To see that (b) holds, suppose that $A^{\prime} \in[A]^{\omega}$ for each $A \in \mathcal{A}_{2}$. Let $A_{0, \alpha}=\left\{(\alpha, \beta): \beta \in \omega_{2}\right\}$ and $A_{1, \alpha}=\left\{(\beta, \alpha): \beta \in \omega_{2}\right\}$. Fix an uncountable $\alpha<\omega_{2}$ so that $A_{0, \beta}^{\prime} \subseteq \alpha^{2}$ for each $\beta \in \alpha$. Consider $A_{1, \alpha}$. As $\alpha$ is uncountable, we can fix $\beta<\alpha$ so that $(\beta, \alpha) \notin A_{1, \alpha}^{\prime}$. But then also $(\beta, \alpha) \notin A_{0, \beta}^{\prime}$, so the refined family is not point- $<2$.

Assume by induction that $n>2$ and $\mathcal{A}_{k}$ is as required for each $k<n$. Clearly $\mathcal{A}_{n}$ is point- $<n+1$ and almost disjoint. So suppose that $A^{\prime} \in[A]^{\omega}$ for each $A \in \mathcal{A}_{n}$. Fix $\alpha<\omega_{n}$ such that $|\alpha|=\omega_{n-1}$ and so that $A_{s}^{\prime} \subseteq \alpha^{n}$ for each $i \in n$ and each $s: n \backslash\{i\} \rightarrow \alpha$. Let

$$
S=\left\{t \in{ }^{n} \omega_{n}: t(n-1)=\alpha \text { and } t(i) \in \alpha \text { for each } i<n-1\right\} .
$$

For each $i<n-1$, fix $s: n \backslash\{i\} \rightarrow \alpha+1$ such that $s$ is the restriction of some element of $S$ to $n \backslash\{i\}$. Thus $s(n-1)=\alpha$ and $B_{s}=\{t \in S: t$ extends $s\} \subseteq A_{s}$. By the induction hypothesis, $\left\{B_{s} \backslash A_{s}^{\prime}: s=t \mid(n \backslash\{i\})\right.$ for some $t \in S$ and $\left.i<n-1\right\}$ is not point- $<n-1$. Therefore, there is $t \in S$ such that for each $i<n-1$, if $s=t \mid(n \backslash\{i\})$ then $t \in A_{s} \backslash A_{s}^{\prime}$. Finally, since $t \mid(n-1) \in{ }^{n-1} \alpha$ we have $A_{t \mid n-1}^{\prime} \subseteq \alpha^{n}$. Therefore $t \in A_{t \mid(n-1)} \backslash A_{t \mid(n-1)}^{\prime}$, so $\left\{A \backslash A^{\prime}: A \in \mathcal{A}_{n}\right\}$ is not point- $<n$.

By taking $\mathcal{A}=\bigcup_{n \in \omega} \mathcal{A}_{n}$ we obtain a family satisfying the following:

Corollary 4. There is a point-finite, almost disjoint family $\mathcal{A}$ such that for each sequence $\left\{A^{\prime}: A \in \mathcal{A}\right\}$ of countable sets, the family $\left\{A \backslash A^{\prime}: A \in \mathcal{A}\right\}$ is not point $-<n$ for any $n \in \omega$.

We now present the main applications of Theorem 2 and Theorem [3, Letting $\sigma=\tau=\aleph_{0}$ in (1.1), we get: 
Corollary 5. Assume CECA. Suppose that $\left\langle A_{\alpha}\right\rangle_{\alpha<\kappa}$ is a sequence of sets such that every $I \in[\kappa]^{\aleph_{0}}$ has a finite subset $J \subset I$ such that $\left|\bigcap_{\alpha \in J} A_{\alpha}\right|<\aleph_{0}$. Then there are $A_{\alpha}^{\prime} \in\left[A_{\alpha}\right] \leq \aleph_{0}$ for $\alpha<\kappa$ such that the sequence $\left\langle A_{\alpha} \backslash A_{\alpha}^{\prime}\right\rangle_{\alpha<\kappa}$ is point-finite.

Letting $\sigma=\aleph_{0}$ and $\tau=\aleph_{1}$ in (1.1), we get:

Corollary 6. Assume CECA. Suppose that $\left\langle A_{\alpha}\right\rangle_{\alpha<\kappa}$ is a sequence of sets such that for every countably infinite set $S$, the set $\left\{\alpha<\kappa: S \subset A_{\alpha}\right\}$ is countable. Then there are $A_{\alpha}^{\prime} \in\left[A_{\alpha}\right]^{\leq \aleph_{0}}$ for $\alpha<\kappa$ such that the sequence $\left\langle A_{\alpha} \backslash A_{\alpha}^{\prime}\right\rangle_{\alpha<\kappa}$ is point-countable.

Let us restate the first part of Theorem 3 in a more conventional way:

Corollary 7. Assume CECA. Let $\sigma$ be a regular infinite cardinal. Suppose that $\left\langle A_{\alpha}\right\rangle_{\alpha<\kappa}$ is a sequence of sets of cardinality $\sigma^{+}$, each such that $A_{\alpha} \cap A_{\beta}$ has cardinality less than $\sigma$ for all $\alpha<\beta<\kappa$. Then there are $A_{\alpha}^{\prime} \in\left[A_{\alpha}\right] \leq \sigma$ for $\alpha<\kappa$ such that the sequence $\left\langle A_{\alpha} \backslash A_{\alpha}^{\prime}\right\rangle_{\alpha<\kappa}$ consists of pairwise disjoint sets.

The consistency of Corollary 7 was already derived from a statement similar to CECA as Theorem 2.6 in 3 .

Condition (1.4) allows us in certain circumstances to relax the assumption that the intersection of each pair of sets $A_{\alpha}, A_{\beta}$ has cardinality strictly less than $\sigma$. In particular, if $\sigma=\aleph_{0}$, then we get

Corollary 8. Assume CECA. Suppose that $\left\langle A_{\alpha}\right\rangle_{\alpha<\kappa}$ is a sequence of sets of cardinality $\aleph_{1}$ such that for every countably infinite set $S$, the set $\left\{\alpha<\kappa: S \subset A_{\alpha}\right\}$ is countable and $\left|A_{\alpha} \cap A_{\beta}\right| \leq \aleph_{0}$ for $\alpha<\beta<\kappa$. Then there are $A_{\alpha}^{\prime} \in\left[A_{\alpha}\right] \leq \aleph_{0}$ for $\alpha<\kappa$ such that the sequence $\left\langle A_{\alpha} \backslash A_{\alpha}^{\prime}\right\rangle_{\alpha<\kappa}$ consists of pairwise disjoint sets.

If the existence of certain large cardinals is consistent, none of the above corollaries is a consequence of GCH alone. To see this, suppose that $\left\langle A_{\alpha}\right\rangle_{\alpha<\kappa}$ is a sequence of sets for which there are $A_{\alpha}^{\prime} \in\left[A_{\alpha}\right]^{\aleph_{0}}$ such that the sequence $\left\langle A_{\alpha} \backslash A_{\alpha}^{\prime}\right\rangle_{\alpha<\kappa}$ is point-countable. Then one can recursively construct for each $\xi<\kappa$ pairwise disjoint sets $B_{\xi} \in[\kappa]^{\aleph_{0}}$ and pairwise disjoint sets $C_{\xi} \subseteq \bigcup_{\alpha \in B_{\xi}} A_{\alpha} \backslash A_{\alpha}^{\prime}$ such that $C_{\xi} \cap A_{\alpha} \backslash A_{\alpha}^{\prime}$ is infinite for each $\alpha \in B_{\xi}$. Now it is easy to construct a function $c:\left[\bigcup_{\alpha<\kappa} A_{\alpha}\right]^{2} \rightarrow\{0,1\}$ such that $\left|c\left(\left[A_{\alpha}\right]^{2}\right)\right|=2$ for all $\alpha<\kappa$. But, if the existence of a supercompact cardinal is consistent, one can construct a model of ZFC where GCH holds and where there exists a sequence $\left\langle A_{\alpha}\right\rangle_{\alpha<\aleph_{\omega+1}}$ of sets of size $\aleph_{1}$ each such that every two of these sets have finite intersection, and for every function $c: \bigcup_{\alpha<\aleph \omega+1} A_{\alpha} \rightarrow\{0,1\}$ there exists $\alpha$ with $\left|c\left[A_{\alpha}\right]\right|=1$ (see Theorem 4.6 of [3]).

However, assuming only GCH, we can get weaker versions of Theorem 2 that are also of interest. For example:

Theorem 4. Suppose that $\sigma$ is a regular infinite cardinal and assume that $\lambda^{\sigma}=\lambda$ for each cardinal $\lambda$ of cofinality greater than $\sigma$. Suppose that $\bar{A}=\left\langle A_{\alpha}\right\rangle_{\alpha<\kappa}$ is a sequence of (not necessarily distinct) sets such that

(*) For every $I \in[\kappa]^{\aleph_{0}}$ there is a finite $J \subseteq I$ such that $\left|\bigcap_{\alpha \in J} A_{\alpha}\right| \leq \sigma$.

Then there exists $\left\langle A_{\alpha}^{\prime}\right\rangle_{\alpha<\kappa}$ such that $\left|A_{\alpha}^{\prime}\right| \leq \sigma^{+}$for each $\alpha<\kappa$ and the sequence $\left\langle A_{\alpha} \backslash A_{\alpha}^{\prime}\right\rangle_{\alpha<\kappa}$ is point-finite.

Proof. Assume by induction that $\kappa$ is minimal such that there is a counterexample to the theorem.

Case 1: $\sigma^{+} \geq \kappa$. Fix an elementary submodel $M$ of size $\sigma^{+} \geq \kappa$ such that $\bar{A} \cup\{\bar{A}\} \cup \sigma^{+} \cup\left\{\sigma^{+}\right\} \subseteq M$. For each $\alpha \in \kappa$ let $A_{\alpha}^{\prime}=A_{\alpha} \cap M$. To see that 
$\left\langle A_{\alpha} \backslash A_{\alpha}^{\prime}\right\rangle_{\alpha<\kappa}$ is point-finite, fix $x \notin M$ such that $I=\left\{\alpha: x \in A_{\alpha}\right\}$ is infinite. By (*), fix a finite $J \subseteq I$ such that $\left|\bigcap_{\alpha \in J} A_{\alpha}\right| \leq \sigma$. Clearly $\bigcap_{\alpha \in J} A_{\alpha} \in M$. But $\sigma^{+} \subseteq M$ implies that $\bigcap_{\alpha \in J} A_{\alpha} \subseteq M$, contradicting $x \notin M$.

Case 2: $\sigma^{+}<\kappa$. If $\kappa=\lambda^{+}$for $\lambda$ a cardinal of cofinality $\leq \sigma$, we fix a continuous $\in$-chain $\left\{M_{\xi}: \xi<\kappa\right\}$ of elementary submodels of some $H(\Theta)$ such that

(a) $\left|M_{\xi}\right|=\lambda$ and $\lambda \subseteq M_{\xi}$ for each $\xi \in \kappa$,

(b) $\bar{A} \in M_{0}$ and $\bar{A} \subseteq \bigcup_{\xi \in \kappa} M_{\xi}$, and

(c) for $\xi$ a successor ordinal we have $M_{\xi}=\bigcup_{i<c f(\lambda)} M_{\xi}^{i}$, where, for each $i$,

(i) $\sigma<\left|M_{\xi}^{i}\right|<\lambda$ and

(ii) $\left[M_{\xi}^{i}\right]^{\sigma} \subseteq M_{\xi}^{i+1}$.

Clearly by our assumption such a sequence exists.

In the case that $\kappa$ is not a successor of a singular cardinal of cofinality $\leq \sigma$ we may fix a sequence as above with the stronger property that $\left[M_{\xi}\right]^{\sigma} \subseteq M_{\xi}$ for each successor $\xi$.

Notice that by $(*)$ we have that, for any model $M$ as above and for any $x \notin M$, $\left\{\alpha \in M: x \in A_{\alpha}\right\}$ is finite. Indeed, if not, we can fix a finite subset $J$ for which $\left|\bigcap_{\alpha \in J} A_{\alpha}\right| \leq \sigma$. But then $S=\bigcap_{\alpha \in J} A_{\alpha}$ is an element of $M$, but it is not a subset. This implies that $|S| \geq \lambda>\sigma$, a contradiction.

The main lemma we need is the following.

Lemma 9. $\left|A_{\alpha} \cap M_{\xi}\right| \leq \sigma^{+}$for each $\xi<\kappa$ and each $\alpha \notin M_{\xi}$.

Proof. Suppose not. First consider the case that $\xi$ is a successor. Then there is an $i<c f(\lambda)$ such that $\left|A_{\alpha} \cap M_{\xi}^{i}\right|>\sigma^{+}$. Therefore there is an $S \in\left[A_{\alpha} \cap M_{\xi}^{i}\right]^{\sigma}$ such that $S \in M_{\xi}^{i+1} \subset M_{\xi}$. But then $\left\{\beta: S \subseteq A_{\beta}\right\} \in M_{\xi}$, but it is not a subset. Therefore it is infinite, contradicting $(*)$. In the case that $\xi$ is a limit, consider two subcases: If $c f(\xi)=\sigma^{++}$, then there is a successor $\eta<\xi$ such that $\left|A_{\alpha} \cap M_{\eta}\right| \geq \sigma^{+}$. If $c f(\xi) \neq \sigma^{++}$, then there is a successor $\eta<\xi$ such that $\left|A_{\alpha} \cap M_{\eta}\right| \geq \sigma^{++}$. Reasoning as above, we get a contradiction to $(*)$ in both cases.

Now, to complete the proof of the theorem, using the lemma and our inductive assumption. Fix $A_{\alpha}^{\prime} \in\left[A_{\alpha}\right] \leq \sigma^{+}$for each $\xi \in \kappa$ and $\alpha \in M_{\xi+1} \backslash M_{\xi}$ such that

(d) $\left\{A_{\alpha} \backslash A_{\alpha}^{\prime}: \alpha \in M_{\xi+1} \backslash M_{\xi}\right\}$ is point-finite, and

(e) $A_{\alpha} \cap M_{\xi} \subseteq A_{\alpha}^{\prime}$.

The proof that $\left\{A_{\alpha} \backslash A_{\alpha}^{\prime}: \alpha \in \kappa\right\}$ is point-finite is now straightforward: Fix any $x \in \bigcup \bar{A}$. If $x \notin \bigcup_{\xi} M_{\xi}$, then consider $I=\left\{\alpha: x \in A_{\alpha}\right\}$. If $I$ is infinite, fix a finite $J \subseteq I$ such that $\left|\bigcap_{\alpha \in J} A_{\alpha}\right| \leq \sigma$. Then there is a $\xi$ such that $J \subseteq M_{\xi}$. But then $S=\bigcap_{\alpha \in J} A_{\alpha} \in M_{\xi}$ and $S \subseteq M_{\xi}$, since $|S| \leq \sigma$, contradicting $x \in S \backslash M_{\xi}$.

If $\xi$ is such that $x \in M_{\xi+1} \backslash M_{\xi}$, then by our construction $\left\{\alpha: x \in A_{\alpha}\right\} \subseteq M_{\xi}$ must be finite as above.

A slightly more general version of Theorem 4 with a slightly weaker conclusion also follows from GCH alone. We don't know whether the following theorem follows from the weaker assumptions given in Theorem 4

Theorem 5. Assume $\mathrm{GCH}$, and let $\sigma, \tau$ be regular infinite cardinals. Suppose that $\bar{A}=\left\langle A_{\alpha}\right\rangle_{\alpha<\kappa}$ is a sequence of (not necessarily distinct) sets such that

(*) For every $I \in[\kappa]^{\tau}$ there is $J \in[I]^{<\tau}$ such that $\left|\bigcap_{\alpha \in J} A_{\alpha}\right| \leq \sigma$.

Then there exist $\left\langle A_{\alpha}^{\prime}\right\rangle_{\alpha<\kappa}$ such that $\left|A_{\alpha}^{\prime}\right| \leq \sigma^{+}$for each $\alpha<\kappa$ and the sequence $\left\langle A_{\alpha} \backslash A_{\alpha}^{\prime}\right\rangle_{\alpha<\kappa}$ is point $-<\tau^{+}$. 
Proof. We modify the proof of Theorem 4 First note that we may assume that $\sigma^{+}, \tau<\kappa$ and that $\kappa$ is the successor of a limit cardinal $\lambda$ of cofinality $\leq \sigma$. Fix $M_{\xi}$ satisfying (a), (b) and (c) of the previous proof. In addition, assume

$\left(\mathrm{c}^{\prime}\right)\left[M_{\xi}^{i}\right] \leq \tau \subseteq M_{\xi}^{i+1}$, for each successor $\xi<\kappa$ and each $i<c f(\lambda)$.

Define $A_{\alpha}^{\prime}$ as before, also such that $\left\langle A_{\alpha} \backslash A_{\alpha}^{\prime}: \alpha \in M_{\xi+1} \backslash M_{\xi}\right\rangle$ is point- $<\tau^{+}$. To see that the whole sequence $\left\langle A_{\alpha} \backslash A_{\alpha}^{\prime}\right\rangle_{\alpha<\kappa}$ is point- $<\tau^{+}$, fix $a \in \bigcup_{\alpha<\kappa} A_{\alpha}$ and fix $\xi$ maximal so that $a \notin M_{\xi}$ (where $M_{\kappa}=\bigcup_{\xi \in \kappa} M_{\xi}$ ). It suffices to prove

Lemma 10. $I=\left\{\alpha \in M_{\xi}: a \in A_{\alpha}\right\}$ has cardinality $<\tau^{+}$.

Proof. Suppose not.

Case 1: $c f(\lambda) \neq \tau$. Then there are a successor $\eta<\xi$ and an $i<c f(\lambda)$ such that $\left|I \cap M_{\eta}^{i}\right| \geq \tau$. Fix $J \in\left[I \cap M_{\eta}^{i}\right]^{<\tau}$ such that $\left|\bigcap_{\alpha \in J} A_{\alpha}\right| \leq \sigma$. By (f), $J \in M_{\eta}$. Therefore $\bigcap_{\alpha \in J} A_{\alpha} \in M_{\eta}$. Therefore $\bigcap_{\alpha \in J} A_{\alpha} \subseteq M_{\eta}$, since it is of size $\leq \sigma$. This contradicts $a \notin M_{\xi}$.

Case 2: $c f(\lambda)=\tau$. In the case that $c f(\xi) \neq \tau^{+}$we can find a successor $\eta<\xi$ such that $\left|I \cap M_{\eta}^{i}\right| \geq \tau^{+}$. This gives a contradiction as in Case 1. Otherwise assume that $c f(\xi)=\tau^{+}$. First fix a successor $\eta$ such that $\left|I \cap M_{\eta}\right| \geq \tau$. Then fix $J \in\left[I \cap M_{\eta}\right]^{<\tau}$ such that $\left|\bigcap_{\alpha \in J} A_{\alpha}\right| \leq \sigma$. Now find $i<c f(\lambda)=\tau$ such that $J \subseteq M_{\eta}^{i}$. So $J \in M_{\eta}$ giving a contradiction as before.

Taking $\sigma=\aleph_{0}$ in Theorem G gives the following.

Corollary 9. Assume $\lambda^{\omega}=\lambda$ for each cardinal $\lambda$ of uncountable cofinality. Suppose that $\left\langle A_{\alpha}\right\rangle_{\alpha<\kappa}$ is a sequence of sets such that $\left|A_{\alpha} \cap A_{\beta}\right| \leq \aleph_{0}$ for each pair of distinct $\alpha, \beta \in \kappa$. Then there are $A_{\alpha}^{\prime} \in\left[A_{\alpha}\right]^{\leq \aleph_{1}}$ for $\alpha<\kappa$ such that the sequence $\left\langle A_{\alpha} \backslash A_{\alpha}^{\prime}\right\rangle_{\alpha<\kappa}$ is point-finite.

Finally, we remark that Theorem 2 cannot be improved by demanding that the sets $A_{\alpha}^{\prime}$ be of cardinality $<\sigma$. Indeed, if $\sigma$ is regular, then let $\left\{A_{\alpha}: \alpha<\sigma^{+}\right\}$be a family of subsets of $\sigma$ such that $\left|A_{\alpha} \cap A_{\beta}\right|<\sigma$ while $\left|A_{\alpha}\right|=\sigma$ for each $\alpha<\sigma^{+}$. No such family can be point- $<\sigma^{+}$. So no point $-<\sigma^{+}$family can be obtained by deleting sets of size less than $\sigma$ from each $A_{\alpha}$.

\section{TOPOLOGICAL APPLICATIONS}

In this section, we present some topological applications of the results just proved. We are particularly interested in the question, raised in 4, of whether a first countable space with a weakly uniform base must have a point-countable base.

Definition 3. If $n \in \omega$ and $\mathcal{B}$ is a base for a topological space $X$, then we say $\mathcal{B}$ is an $n$-weakly uniform base for $X$ provided that if $A \subseteq X$ with $|A|=n$, then $\{B \in \mathcal{B}: A \subseteq B\}$ is finite. We say a base $\mathcal{B}$ for $X$ is a $<\omega$-weakly uniform base provided that if $A \subseteq X$ with $|A| \geq \aleph_{0}$, then there is a finite subset $F \subseteq A$ with $\{B \in \mathcal{B}: F \subseteq B\}$ finite.

The notion of weakly uniform base, introduced in [4], is just what we have called a 2-weakly uniform base. We shall see later in this section that the properties defined above are all distinct. To avoid trivialities we will be interested only in the case $n \geq 2$. Clearly, for $n<m, n$-weakly uniform, base implies $m$-weakly uniform base which implies $<\omega$-weakly uniform base. 
It was shown in 4 that if $X$ is a space with a 2-weakly uniform base $\mathcal{B}$ and $x \in X$ is in the closure of a countable subset of $X \backslash\{x\}$, then $\mathcal{B}$ is point-countable at $x$. In particular, if $X$ is a first countable space with a weakly uniform base $\mathcal{B}$, then $\mathcal{B}$ is point-countable at all nonisolated points of $X$. Using that result, it was shown in [4 that a first countable space in which the boundary of the set of isolated points is separable has a point-countable base.

In [2], it is shown that a first countable space with a weakly uniform base and no more than $\aleph_{1}$ isolated points has a point-countable base. In [1] this is improved (consistently) to the result that, assuming $\mathrm{CH}$, every first countable space with a weakly uniform base and no more than $\aleph_{\omega}$ isolated points has a point-countable base.

An example is constructed in [2, assuming MA and $\aleph_{2}<2^{\aleph_{0}}$, of a normal Moore space with a weakly uniform base which has no point-countable base. Such a space also could not be meta-Lindelöf.

Our next result establishes the independence of the existence of first countable spaces with weakly uniform bases but without point-countable bases, and it removes the cardinality restriction on the set of isolated points.

Theorem 6. Assume CECA. If $X$ is a $T_{1}$-space in which each non-isolated point is a cluster point of a countable set and $X$ has a $<\omega$-weakly uniform base, then $X$ has a point-countable base.

Proof. Suppose $\mathcal{B}$ is a $<\omega$-weakly uniform base for $X$. Suppose $x$ is a non-isolated point of $X$. We will show that $\mathcal{B}$ is point-countable at $x$. Choose a countable set $C \subseteq X \backslash\{x\}$ with $x \in \bar{C}$. For each $B \in \mathcal{B}$ with $x \in B$, the set $C \cap B$ is infinite, and hence there exists $F_{B} \in[C \cap B]^{<\aleph_{0}}$ such that $\left\{A \in \mathcal{B}: F_{B} \subset A\right\}$ is finite. Since $[C]^{<\aleph_{0}}$ is countable and for each $F \in[C]^{<\aleph_{0}}$ there are only finitely many $B \in \mathcal{B}$ with $F_{B}=F$, we conclude that $\{B \in \mathcal{B}: x \in B\}$ is countable.

Let $\left\langle x_{\alpha}: \alpha<\kappa\right\rangle$ list the isolated points of $X$, and let $A_{\alpha}=\left\{B \in \mathcal{B}: x_{\alpha} \in B\right\}$. Notice that since $\mathcal{B}$ is a $<\omega$-weakly uniform base, we have that if $I$ is an infinite subset of $\kappa$, then there exists a finite $J \subseteq I$ such that

$$
\left|\left\{B \in \mathcal{B}:\left\{x_{\alpha}: a \in J\right\} \subseteq B\right\}\right|<\aleph_{0},
$$

i.e. $\left|\bigcap_{\alpha \in J} A_{\alpha}\right|<\aleph_{0}$. By Corollary 5, for each $\alpha$ there is $A_{\alpha}^{\prime} \in\left[A_{\alpha}\right] \leq \aleph_{0}$ such that $\left\langle A_{\alpha} \backslash A_{\alpha}^{\prime}: \alpha<\kappa\right\rangle$ is point-finite, i.e. for each $B \in \mathcal{B}$ we have $B \in A_{\alpha} \backslash A_{\alpha}^{\prime}$ for only finitely many $\alpha$ 's. Hence for each $B \in \mathcal{B}$ there is a finite set $I(B)$ of isolated points such that, for every $\alpha<\kappa,\left\{B \in \mathcal{B}: x_{\alpha} \in B \backslash I(B)\right\} \subseteq A_{\alpha}^{\prime}$, and $\left|A_{\alpha}^{\prime}\right| \leq \aleph_{0}$. Hence $\mathcal{B}_{1}=\{B \backslash I(B): B \in \mathcal{B}\}$ is a point-countable open family of subsets of $X$ which forms a base at every non-isolated point of $X$. Thus $\mathcal{B}^{*}=\mathcal{B}_{1} \cup\left\{\left\{x_{\alpha}\right\}: \alpha<\kappa\right\}$ is a point-countable base for $X$.

Corollary 10. It is consistent with ZFC that every first countable $T_{1}$-space with a weakly uniform base has a point-countable base.

Combining that corollary with the example of [2] completes the independence result.

Assuming only $\lambda^{\omega}=\lambda$ for each cardinal $\lambda$ of uncountable cofinality, we can obtain a weaker version of Theorem [6. The proof is identical, using Corollary 9 in place of Corollary 5 
Theorem 7. Assume $\lambda^{\omega}=\lambda$ for each cardinal $\lambda$ of uncountable cofinality. If $X$ is a $T_{1}$-space in which each non-isolated point is a cluster point of a countable set and $X$ has $a<\omega$-weakly uniform base, then $X$ has a point $-<\aleph_{2}$ base.

Corollary 11. Assume $\lambda^{\omega}=\lambda$ for each cardinal $\lambda$ of uncountable cofinality. Every first countable $T_{1}$-space with a weakly uniform base has a point- $<\aleph_{2}$ base.

Note that if we weaken the conclusion of $<\omega$ weakly uniform base to " $\{B \in$ $\mathcal{B}: F \subseteq B\}$ is countable", then we obtain a notion that implies point-countable at nonisolated points and gives both Theorem 7 and its corollary. It would be interesting to know when this weaker notion sufficed to conclude that the space has a point-countable base.

Definition 4. If $n \in \omega$, then we say that a topological space $X$ is n-metacompact provided that for every open cover $\mathcal{U}$ of $X$ there is an open refinement $\mathcal{V} \prec \mathcal{U}$ such that if $A \subseteq X$ with $|A|=n$, then $\{V \in \mathcal{V}: A \subseteq V\}$ is finite. We say $X$ is $<\omega$-metacompact provided that, for every open cover $\mathcal{U}$ of $X$ there is an open refinement $\mathcal{V} \prec \mathcal{U}$ such that if $A \subseteq X$ with $|A| \geq \aleph_{0}$, then there is a finite subset $F \subseteq A$ with $\{V \in \mathcal{V}: F \subseteq V\}$ finite.

It is clear that 1-metacompact is just metacompact and that, for $n<m, n$ metacompact implies $m$-metacompact, which implies $<\omega$-metacompact. We will call a refinement $\mathcal{V}$ as in the definition above an $n$-weakly uniform refinement, or $<\omega$-weakly uniform refinement, respectively.

Theorem 8. Assume CECA. If $X$ is a $<\omega$-metacompact $T_{1}$-space, then $X$ is meta-Lindelöf.

Proof. Suppose $X$ is $<\omega$-metacompact and $\mathcal{U}$ is an open cover of $X$. Let $\mathcal{V}$ be an open, $<\omega$-weakly uniform refinement of $\mathcal{U}$. List the points of $X$ as $\left\langle x_{\alpha}: \alpha<\kappa\right\rangle$. For each $\alpha<\kappa$, let $A_{\alpha}=\left\{V \in \mathcal{V}: x_{\alpha} \in V\right\}$. By Corollary [5 for each $\alpha<\kappa$ there is $A_{\alpha}^{\prime} \in\left[A_{\alpha}\right] \leq \aleph_{0}$ such that $\left\langle A_{\alpha} \backslash A_{\alpha}^{\prime}: \alpha \in \kappa\right\rangle$ is point-finite on the set $\mathcal{V}$. For each $V \in \mathcal{V}$, we let $I(V)=\left\{x_{\alpha} \in V: V \in A_{\alpha} \backslash A_{\alpha}^{\prime}\right\}$. Note that $|I(V)|<\aleph_{0}$. Further, for each $\alpha<\kappa$ we have $\left\{V \in \mathcal{V}: x_{\alpha} \in V \backslash I(V)\right\} \subseteq A_{\alpha}^{\prime}$ and $\left|A_{\alpha}^{\prime}\right| \leq \aleph_{0}$. For each $\alpha<\kappa$, choose $V_{\alpha} \in \mathcal{V}$ such that $x_{\alpha} \in V_{\alpha}$. For $V \in \mathcal{V}$, let

$$
V^{*}=(V \backslash I(V)) \cup\left\{x_{\alpha} \in I(V): V=V_{\alpha}\right\} \text {. }
$$

Note that $V^{*} \subseteq V$ and $V \backslash V^{*}$ is finite. Let $\mathcal{V}^{*}=\left\{V^{*}: V \in \mathcal{V}\right\}$. Then $\mathcal{V}^{*}$ is an open refinement of $\mathcal{U}$, and if $x_{\alpha} \in V^{*}$, then $V \in A_{\alpha}^{\prime} \cup\left\{V_{\alpha}\right\}$. Thus $\mathcal{V}^{*}$ is point-countable.

Corollary 12. It is consistent with $Z F C$ that every $T_{1}$-space with a weakly uniform base is meta-Lindelöf.

Again, combining this corollary with the example in [2] completes the independence result.

Example 2. For each natural number $n \geq 1$, there is a Moore space $X_{n}$ of scattered height 2 which has an $(n+1)$-weakly uniform base, but $X_{n}$ does not have an $n$-weakly uniform base.

Proof. Let $L$ be a subset of $\mathbf{R} \times\{0\}$ with $|L|=\aleph_{1}$, and let $D=\left\{p_{n}: n \in \omega\right\}$ be a countable subset of $\mathbf{R} \times(0, \infty)$ which is dense in the Euclidean topology. It is shown in [2] that there is a collection $\mathcal{H}$ of countably infinite subsets of $L$ and a partition $\left\{\mathcal{H}_{n}: n \in \omega\right\}$ of $\mathcal{H}$ such that 
(1) if $H_{1}, H_{2} \in \mathcal{H}$ and $H_{1} \neq H_{2}$, then $\left|H_{1} \cap H_{2}\right|<\aleph_{0}$, and

(2) if $Y \subseteq L$ and $|Y|=\aleph_{1}$, then for each $n \in \omega$, there exists $H \in \mathcal{H}_{n}$ such that $|Y \cap H|=\aleph_{0}$.

For each $n \in \omega$, let $Y_{n}=\left\{\left(p_{n}, H\right): H \in \mathcal{H}_{n}\right\}$, and let $Y=\bigcup_{n \in \omega} Y_{n}$. Let $X=L \cup Y$. For each $n \in \omega$ and each $x \in L$, let $B_{n}(x)=\left\{\left(p_{i}, H\right):\left(p_{i}, H\right) \in Y_{i}\right.$, $x \in H$, and $p_{i}$ is an element of the Euclidean open ball in $\mathbf{R} \times(0, \infty)$ of radius $2^{-n}$ which is tangent to the axis $\mathbf{R} \times\{0\}$ at the point $x\}$. If $x \in L$ and $n \in \omega$, then we define $G_{n}(x)=\{x\} \cup B_{n}(x)$ and let $\left\{G_{n}(x): n \in \omega\right\}$ be a neighborhood base at $x$. If $y \in Y$, then $\{y\}$ is open. It is shown in [2] that with this topology $X$ is a Moore space with a weakly uniform base, and if $\mathcal{U}$ is any open cover of $X$ which refines the open cover $\left\{G_{0}(x): x \in L\right\} \cup\{\{y\}: y \in Y\}$, then there exists $y \in Y$ such that $\{U \in \mathcal{U}: y \in U\}$ is infinite.

Suppose $k$ is a natural number and $k \geq 1$. We shall use the space $X$ to construct a Moore space $X_{k}$ of scattered height 2 with a $(k+1)$-weakly uniform base but no $k$-weakly uniform base. Let $X_{k}=L \cup(Y \times k)$. Points of $Y \times k$ will be isolated. If $x \in L$ and $n \in \omega$, let $G_{n}^{k}(x)=\{x\} \cup\left(B_{n}(x) \times k\right)$. Notice that $\left\langle G_{n}^{k}(x): n \in \omega\right\rangle$ is a decreasing sequence, and thus this is a valid assignment of neighborhoods. Letting $\mathcal{G}_{n}=\left\{G_{n}^{k}(x): x \in L\right\} \cup\{\{y\}: y \in Y \times k\}$, we see that $\left\langle\mathcal{G}_{n}: n \in \omega\right\rangle$ is a development for $X_{k}$, and since each $G_{n}^{k}(x)$ is clopen, $X_{k}$ is a 0 -dimensional space of scattered height 2 .

We now show that $\mathcal{B}=\bigcup_{n \in \omega} \mathcal{G}_{n}$ is a $(k+1)$-weakly uniform base for $X_{k}$. Suppose $A \subseteq X_{k}$ with $|A|=k+1$. If $|A \cap L| \geq 2$, then $|\{B \in \mathcal{B}: A \subseteq B\}|=0$. If $|A \cap L|=1$, then let $\{x\}=A \cap L$. Choose $n \in \omega$ such that $G_{n}^{k}(x) \cap A=\{x\}$. Then $|\{B \in \mathcal{B}: A \subseteq B\}| \leq n$, since for each $m \in \omega$ there is only one element of $\mathcal{G}_{m}$ which contains $x$. Finally, if $|A \cap L|=0$, then let $A=\left\{\left(y_{1}, n_{1}\right), \ldots,\left(y_{k+1}, n_{k+1}\right)\right\}$, where $y_{i} \in Y$ and $n_{i} \in k$ for $1 \leq i \leq k+1$. Choose $i, j \leq k+1$ such that $n_{i}=n_{j}$. Then $\{B \in \mathcal{B}: A \subseteq B\} \subseteq\left\{B \in \mathcal{B}:\left\{\left(y_{i}, n_{i}\right),\left(y_{j}, n_{j}\right)\right\} \subseteq B\right\}$. Now for $B=G_{n}^{k}(x)$, we have that $\left(y_{j}, n_{j}\right) \in G_{n}^{k}(x)$ implies $y_{i} \in G_{n}(x)$. So

$$
\left|\left\{B \in \mathcal{B}:\left\{\left(y_{i}, n_{i}\right),\left(y_{j}, n_{j}\right)\right\} \subseteq B\right\}\right|=\left|\left\{G_{n}(x):\left\{y_{i}, y_{j}\right\} \subseteq G_{n}(x)\right\}\right|<\aleph_{0},
$$

since $\left\{G_{n}(x): n \in \omega, x \in L\right\}$ is shown in [2] to be a weakly uniform collection.

We now show that $X_{k}$ has no $k$-weakly uniform base. Suppose $\mathcal{V}$ is any open cover of $X_{k}$ which refines $\mathcal{G}_{1}$. For each $x \in L$, choose $V_{x} \in \mathcal{V}$ and $n(x) \in \omega$ with $G_{n(x)}^{k}(x) \subseteq V_{x}$. Now in the space $X, \mathcal{U}=\left\{G_{n(x)}(x): x \in L\right\} \cup\{\{y\}: y \in Y\}$ refines $\left\{G_{1}(x): x \in L\right\} \cup\{\{y\}: y \in Y\}$. Hence there is a point $y \in Y$ so that $\{U \in \mathcal{U}: y \in U\}$ is infinite, and so $\left\{x \in L: y \in G_{n(x)}(x)\right\}$ is infinite. Let $A=\{y\} \times k$. Then $|A|=k$, and for each $i \in k$ we have $(y, i) \in G_{n(x)}^{k}(x) \subseteq V_{x}$. So $\left\{x \in L: A \subseteq V_{x}\right\}$ is infinite, and thus $\mathcal{V}$ is not a $k$-weakly uniform base for $X_{k}$.

Example 3. There is a space $Y$ which has $<\omega$-weakly uniform base, but does not have an n-weakly uniform base for any $n \in \omega$.

Proof. For each natural number $n \geq 1$, let $X_{n}$ be as constructed in Example 2, Let $Y$ be the disjoint union of the spaces $X_{n}$. The natural base is easily seen to be $<\omega$-weakly uniform, since if $A \subseteq Y$ and $|A|=\aleph_{0}$, then either $A \subseteq X_{n}$ for some $n$, in which case any subset of $A$ of size $n+1$ would be contained in only finitely many elements of the base, or $A$ contains two points from distinct $X_{n}$ 's, in which case no element of the base contains that two-point subset. To see that $Y$ cannot have an $n$-weakly uniform base, it is enough to observe that $X_{n}$ is an open subspace of $Y$. 
Thus we have shown that these properties are all distinct.

\section{REFERENCES}

[1] A. V. Arhangel'skii, W. Just, E. A. Reznichenko, and P. J. Szeptycki; Sharp bases and weakly uniform bases versus point-countable bases, to appear in Topology and its Applications.

[2] S. W. Davis, G. M. Reed, and M. L. Wage; Further results on weakly uniform bases, Houston Journal of Mathematics, 2 (1976) 57-63. MR 52:15365

[3] A. Hajnal, I. Juhász, and S. Shelah [HJSh249]; Splitting strongly almost disjoint families, TAMS 295 (1986) 369-387. MR 87i:03098

[4] R. W. Heath and W. F. Lindgren; Weakly uniform bases, Houston J. Math. 2 (1976) 85-90. MR 52:15364

[5] S. Shelah [Sh108]; On Successors of Singular Cardinals, in: Logic Colloquium 78 (M. Boffa, D. van Dalen, K. McAloon, eds.), 357-380, North-Holland 1979. MR 82d:03079

[6] S. Shelah [Sh88a]; Appendix: On stationary sets (to "Classification of nonelementary classes II. Abstract elementary classes"), in: Classification Theory, Proceedings of the USA-Israel Conference on Classification Theory, Chicago, December 1985, J. T. Baldwin, ed., LNM 1292, 483-495, Springer 1987. MR 91h:03046

[7] S. Shelah [Sh420]; Advances in Cardinal Arithmetic, Finite and Infinite Combinatorics in Sets and Logic (N. W. Sauer et al. eds.), NATO Adv. Sci. Inst. Ser. C: Math. Phys. Sci., vol. 411, Kluwer 1993, 355-383. MR 95h:03112

Department of Mathematics, Miami University, Oxford, Ohio 45056

E-mail address: ztbalogh@miavx1.acs.muohio.edu

Department of Mathematics, Miami University, Oxford, Ohio 45056

E-mail address: swdavis@miavx1.muohio.edu

Department of Mathematics, Ohio University, Athens, Ohio 45701

E-mail address: just@math.ohiou.edu

Institute of Mathematics, The Hebrew University, Givat Ram, 91904 Jerusalem, ISRAEL

E-mail address: shelah@math.huji.ac.il

Department of Mathematics, Ohio University, Athens, Ohio 45701

E-mail address: szeptyck@math.ohiou.edu 